\title{
Automated Topology Correction for Human Brain Segmentation
}

\author{
Lin Chen and Gudrun Wagenknecht \\ Central Institute for Electronics, Research Center Juelich, Juelich, Germany
}

\begin{abstract}
We describe a new method to reconstruct human brain structures from 3D magnetic resonance brain images. Our method provides a fully automatic topology correction mechanism, thus avoiding tedious manual correction. Topological correctness is important because it is an essential prerequisite for brain atlas deformation and surface flattening. Our method uses an axis-aligned sweep through the volume to locate handles. Handles are detected by successively constructing and analyzing a directed graph. A multiple local region-growing process is used which simultaneously acts on the foreground and the background to isolate handles and tunnels. The sizes of handles and tunnels are measured, then handles are removed or tunnels filled based on their sizes. This process was used for 256 T1-weighted MR volumes.
\end{abstract}

\section{Introduction}

Representing the shape of complex anatomical regions such as human brain structures has always been a challenge. Recent advances allow detailed anatomical information to be derived from magnetic resonance imaging (MRI), but due to imaging noise artifacts, partial volume effects and intensity inhomogeneities, automatic extraction of topologically correct brain structures remains difficult, especially for regions in the cortex.

$\mathrm{Xu}$ et al. 1] introduced automatic surface topology correcting which used fuzzy segmentation to produce an isosurface representation of the WM/GM interface. If the surface is not homeomorphic to a sphere, a median filter is used to iteratively smoothen the fuzzy segmentation until a surface with the correct topology is found. One drawback of this approach is that using a $3 \times 3 \times 3$ median filter does not guarantee the correct topology.

Several methods for correcting the topology of the cortical surface have recently been developed. The region growing model proposed by Kriegeskorte et al. [2] is adopted as a topology correction method. It starts from an initial point with the deepest distance to the surface, and then grows the point by adding points that will not change the topology (without self-touching). One drawback of this approach is that the result strongly depends on the order in which the points are grown from the growing points set, and another one is that it considers only foreground without any size criterion. Dale et al. 3] flattened a cortex isosurface by ignoring small handles, identifying and fixing topology defects on the inflated cortical surface, but leaving some large handles to be edited manually. More 
recently, Fischl et al. 4 inflated the cortex isosurface to a sphere and identified the overlapping triangles on the spherical surface which corresponded to handles, then tessellated a new mesh as the topologically correct cortical surface. Shattuck and Leahy [5] and Xiao Han et al. 6] introduced graph-based methods for topology correction. Shattuck and Leahy examined the connectivity of $2 \mathrm{D}$ segmentations between adjoining slices to detect topological defects and minimally correct them by changing as few voxels as possible. One drawback of this approach is that the set of voxels which must be changed to correct the topology are necessarily oriented along the Cartesian axes. This set is henceforth also termed "cut". Building on their work, Han et al. developed an algorithm to remove all handles from a binary object under any connectivity. Successive morphological openings correct the segmentation at the smallest scale. This method is effective for small handles, but large handles may need to be edited manually. Wood et al. [7] proposed a different approach. Handles in the tessellation are localized by simulating wavefront propagation on the tessellation and are detected where the wavefronts meet twice. The size of a handle is the shortest non-separating cut along such a cycle, which helps retain as much fine geometrical detail of the model as possible. One drawback of this method is that it requires the arbitrary vertex tessellation to be converted to a volumetric image in order to correct the defect.

Our method provides a fully automatic topology correction mechanism. The method is similar to Shattuck and Leahy's [5], which is based on connectivity and graphs from sequences of slices. The innovation is that we provide a very quick iterative approach, which shows that corrections need not be oriented along cardinal axes. It can thus be done under any connectivity in $3 D(6,18,26)$ without cutting the whole plane and changing as few voxels as possible by using a threshold.

\section{Background and Definitions}

Some basic concepts of digital topology are outlined in this section. Please refer to $\mathrm{G}$. Bertrand [8] for details. The initial segmentation is a binary digital image $X \in Z^{3}$ composed of a foreground object $F$ and an inverse background object $B=\bar{F}$. According to the conventional definition of adjacency, three types of connectivity might be considered: 6-, 18- and 26-connectivity. In order to avoid topological paradoxes, different connectivities, $n$ and $\bar{n}$, must be used for $F$ and $B$. The following four pairs of compatible connectivities - $(6,26),(6,18),(26,6)$ and $(18,6)$ - are generally used. Considering a digital object, computation of two numbers will suffice to check if modifying one voxel will affect the topology. These topological numbers, denoted $T_{n}$ and $T_{\bar{n}}$, were introduced by Bertrand [8] as an elegant way to classify the topology type of a given voxel which will be used by the algorithm. The object is henceforth termed $X$ and its inverse object $\bar{X}$. Furthermore, the following definitions will also be used:

Definition 1 (Simple point). a point $x \in X$ that can be added or removed without changing the topology of an object. It is characterized by $T_{n}(x, X)=$ $T_{\bar{n}}(x, \bar{X})=1$. 
Definition 2 (Border point). a point $x \in X$ is called a $n$-border point, if and only if at least one of its $n$-neighbor $x^{\prime}, x^{\prime} \in \bar{X}$. It is called a $\bar{n}$-border point, if and only if $x \in \bar{X}$ and at least one of its $\bar{n}$-neighbor $x^{\prime}, x^{\prime} \in X$.

Definition 3 (Residue and body point). The algorithm generates different connected components and assigns voxels with different labels for each component. Residue points characterize voxels belonging to a component that will change the topology of the object. If their component will not change the topology, they are termed body points.

\section{Methods}

A method is described whereby the topology of a volumetric binary image can be altered so that an isosurface algorithm can generate a surface with the topology of a sphere, i.e., its genus is zero. The genus of a surface can be determined by its Euler characteristic [6] which is a topological invariant of the generated isosurface. However, it provides only a global criterion for topology correction, but no information about the position and size of the handles and tunnels. Therefore, the size and the location of both handles and tunnels need to be found.

To that end, the topology of the object is analyzed while also analyzing its geometry. Two directed graphs are formed, one for foreground and one for background voxels which contain information about the volume. Based on the approach described below, this makes it possible to determine whether the foreground object is topologically equivalent to a sphere by counting the number of cycles in the graphs. The cycles also provide information about the size and location of handles or tunnels, namely, if the number of cycles of the foreground graph and background graph is zero, then the generated isosurface of the object has a spherical topology. Our approach is based on this criterion and can be summarized in the following four steps:

1. Sweep through the volume to encode the topology in two directed graphs for foreground and background.

2. Isolate handles using the directed graphs.

3. For each handle found, find the minimum number of voxels (minimal cut) which must be changed to correct the topology.

4. Run iterative multi-axis correction.

\subsection{Connectivity Graph}

A foreground connectivity graph $G=\{N, E\}$ is created which contains important information about the structure of the binary image foreground. The object is examined along a selected cardinal axis, identifying the connected components within each slice perpendicular to the axis, using 2D 4-connectivity if the foreground uses 6-connectivity in 3D, and 2D 8-connectivity if the foreground uses 18- or 26-connectivity in 3D. Each in-slice-connected component is called a node in the graph. Next, it is determined how each node is connected to the nodes 


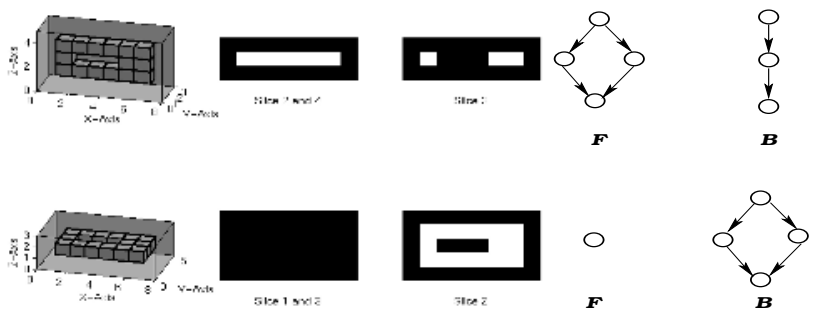

Fig. 1. An $8 \times 3 \times 5$ binary volume with a torus, the corresponding slices and its connectivity graphs for foreground and background

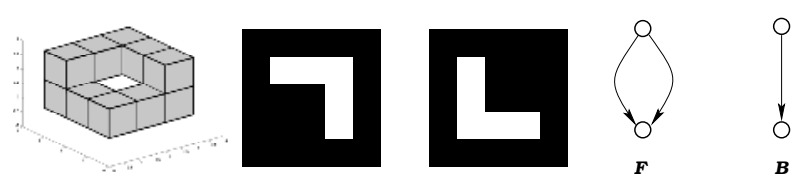

Fig. 2. A $5 \times 5 \times 4$ binary volume with a torus, the corresponding slices and its connectivity graphs for foreground and background

in the next slice. Two nodes are defined as having a directed connection if they are $n$-connected. Each connection in the object is represented by an edge in the graph. Note that one node may be connected to two or more nodes, and that these nodes are connected to one node in the next slice (Fig. 1). In the simplest case, one node will have multiple edges (separate connections) connecting it to one node in the next slice in the foreground. Such a representation is the simplest form of a topological handle existing in the object (Fig. 2). In this case, the handle is detected as the smaller one of these connections. The background connectivity graph is created likewise using $\bar{n}$ connectivity (Fig. 1 and 2).

\subsection{Isolate Handles Using the Directed Graph}

Sweeping through the graph, cycles corresponding to handles are detected successively. The approach is as follows: For each node which is connected with two or more nodes in the next slice, different labels are assigned to the following nodes. A cycle is detected by running a breadth-first search. The way that a cycle can form is when two nodes with these different labels in the previously examined slice are connected to a single node in the next slice (Fig. 3). Note that one cycle has 2 sides with 2 different labels.

When a cycle with two sides is detected, the corresponding handle in the object must be isolated. Choosing the smaller one as the handle comes naturally (Fig. 4 a); i.e., in the real world, the handle of a cup or a tea pot, not the body, is chosen as the handle. Note that a node with $k>2$ subsequent connected nodes may have $k$ child cycles. Since the local genus of the surface is $k-1$, we only need to cut $k-1$ smaller handles to correct the topology. The advantage of thus 


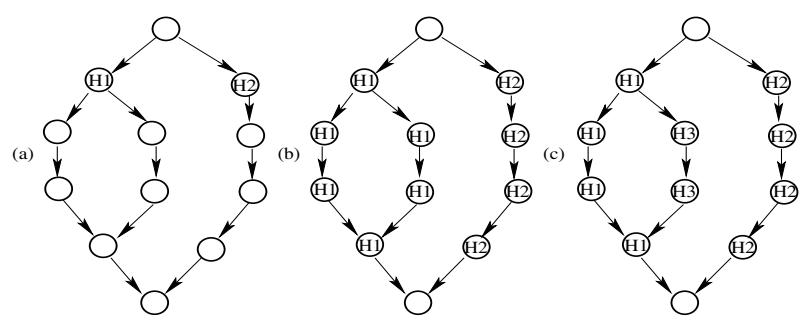

Fig. 3. A sample illustration of handle isolation, from (a) to (c)

choosing handles is that the object need not be separated into several pieces and overlapping cycles.

As per Definition 3, these detected handles divide the object into two kinds of components, the handles consisting of points called residue points, the other points being called body points.

\subsection{Handle Cutting by Cycle Breaking}

The basic idea of topology correction is dividing cycles into body and residue parts and discarding the smallest residue piece in each cycle in 3D. When these voxels are removed from the foreground, they are removed from the object. This is called a handle cut. Conversely, when voxels are removed from the background, they are added to the object, and hence a tunnel is filled.

The handles are the good candidates for these residue parts. However, the thinnest cut of a cycle may not fall within the handle. Hence, we introduce residue part expansion ( $\mathrm{RPE}$ ), which is intended to catch the thinnest cut by iteratively growing the handle. This is done as follows (see Def. 1-3):

\section{Algorithm 1 (Residue Part Expansion) (RPE):}

1. Find the handle set $S$ of residue points that are n-adjacent to the body point set $X$, (Fig. 4 a-b).

2. Find the top and bottom connection sets $X_{1}$ and $X_{2}$, where for each point $x \in X_{1}$ or $x \in X_{2}, x \in X$ and $x$ is n-adjacent to $S$, (Fig. 4 c).

3. Add $X_{1}$ to $\mathrm{S}$.

4. Find the set $T$ which is n-adjacent to $X_{1}$, where for each point $x \in T$, $x \in X \backslash S$ and $x$ is n-adjacent to $X_{1}$.

5. Compare the cardinalities of sets $X_{1}$ and $T$. If $\#\left(X_{1}\right)>\#(T)$, add $T$ to $\mathrm{S}$ and replace $X_{1}$ by $T$, go to step 4 . Otherwise go to next step, (Fig. $4 \mathrm{~d}$ ).

6. Deal with $X_{2}$ in the same way as with $X_{1}$, (steps $\left.3-5\right)$, (Fig. 4 c-d).

7. Deal with $B$ in the same way as with $F$.

Note that after RPE growing, each residual part $S$ is a n-connected component which is n-adjacent to the body part $X$ and a cut of it can reduce the genus by one. This is due to the fact that its growing by border points is equivalent to growing by adding simple points, and so will retain its topology after growing. 


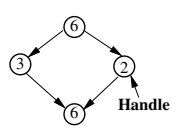

(a)

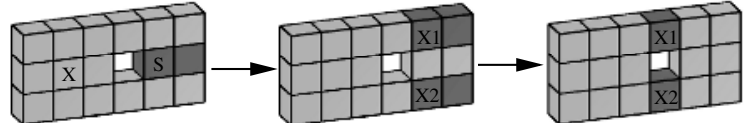

(c)

(d)

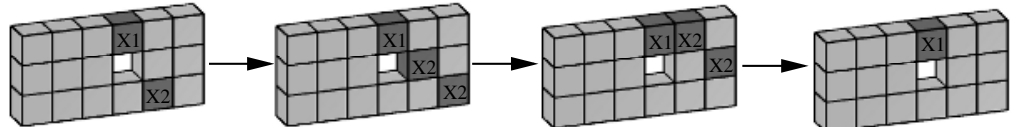

(f) (g) (h)

Fig. 4. Illustration of RPE (a) - (d) and TCB (d) - (h) with 6-connectivity for the foreground

The main idea of cutting the residue part is to transfer as many points as possible from the residue back to the body without changing its topology. Specifically, sets $X_{1}$ and $X_{2}$ are the two cuts of the residue part after RPE, but they might not be the smallest ones. The body set is grown by successively detecting border points of $X_{1}$ or $X_{2}$ which lie within the residue, but only add those points (which are simple points) which come from the residue and do not adversely affect topology (see Def. 1-3). This is done as follows (see Fig. 4 d-h):

\section{Algorithm 2 (Topology cycle breaking) (TCB):}

1. Compare the cardinalities of sets $X_{1}$ and $X_{2}$. If $\#\left(X_{1}\right)>\#\left(X_{2}\right)$, find the set $T$ which is n-adjacent to $X_{1}$, where for each point $x \in T, x \in S \backslash X 1$ and $x$ is n-adjacent to $X_{1}$, add $X_{1}$ to body set $X$. Otherwise go to step 4 .

2. For each point $x \in T$, if $x \in X_{2}$, label $x$ as a cut point, set $\#\left(X_{2}\right)=$ $\#\left(X_{2}\right)-1, \#(T)=\#(T)-1$,

3. if $\#(T)>0$, replace $X_{1}$ by $T$, then go to step 1 . Otherwise go to step 5 .

4. Deal with $X_{2}$ in the same way as with $X_{1}$ (steps 1 - 3).

5. Deal with $B$ in the same way as with $F$.

Note that at each iteration, the largest set of simple points is added to the body. The labeling of cutting points ensures that the final residue points are not added back to the body and are positioned at the thinnest parts of the handles.

\subsection{Iterative Multi-axis Correction}

The nature of the changes described in the previous section depends on the axis along which the object is analyzed. In order to ensure only the smallest changes are made to the object, corrections are applied iteratively along each axis. A threshold is used, and only corrections less than or equal to the threshold are done. This multi-axis approach dramatically reduces the number of voxels which are added to or removed from the object set. The algorithm is outlined below. 


\section{Algorithm 3 (Iterative multi-axis correction) (IMC):}

1. Set the threshold value to one.

2. Starting with the $z$ axis, compute a foreground directed connectivity graph $G=\{N, E\}$.

3. Isolate each handle using the directed graph.

4. Find the handle cutting set $T$ for each handle using RPE and TCB.

5. Remove the handle cutting set from the object if the cardinality of $T$ is less than or equal to the threshold.

6. Repeat steps $2-5$ for the $x$-axis and $y$-axis.

7. Repeat steps $2-6$ for the background.

8. Repeat steps $2-7$ until no further changes can be made at this threshold.

9. Iterate steps $2-8$ with an increasing threshold until no further corrections can be made.

\section{Results}

The topological constraint algorithm was applied to 256 T1-weighted MR volumes. Image size after preprocessing was $160 \times 200 \times 160$ voxels. Processing time for each volume was between 25-86 seconds (average 45 seconds) on an Intel Pentium IV 3.0-GHz CPU without considering the simplest case (Fig. 2). Processing time for each volume was between 4-10 minutes by considering this case, but fewer number of voxels were changed for topology correction. It should be noted that aspects of the implementation of this approach such as frequent graph

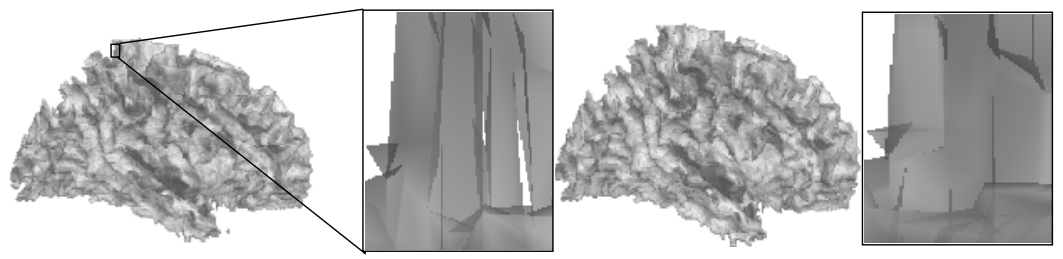

Fig. 5. WM/GM surface before and after topology corrections

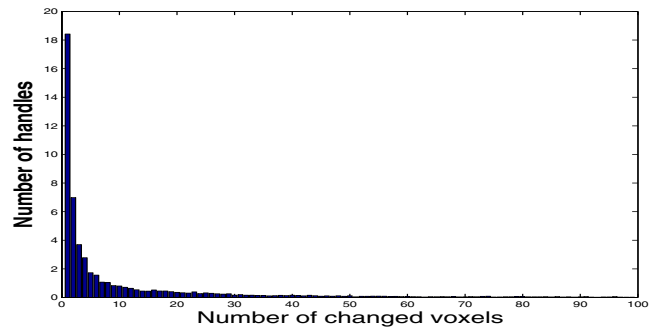

Fig. 6. The histogram shows the average number of handles for the detected handle sizes (thresholds) for the 256 data sets examined 
recalculation can be optimized, providing substantial decreases in computation costs. Each white matter volume was tessellated after topological correction and the genus verified using the Euler-Poincaré formula [6]. In each case, the method produced a final volume with a tessellation having an Euler characteristic of two, corresponding to a genus of zero and homeomorphism to a sphere. Fig. 5 shows one part of a WM/GM surface before and after topology correction. Note that the algorithm was able to correct all volumes without subsampling and with very few changes to the membership set. This algorithm changed between $0.0086 \%$ and $0.202 \%$ of the voxels for each of the 256 volumes, with an average of $0.020 \%$. $94.14 \%$ of the volumes change less than $0.05 \%$ voxels.

\section{Acknowledgements}

We thank Dr. Gabriele Lohmann from Max Planck Institute for Human Cognitive and Brain Sciences who kindly provided us with the brain MR images. Thanks are also due to Alejandro Rodón for language editing.

\section{References}

1. Xu, C., Pham, D.L., Rettmann, M.E., Yu, D.N., Prince, J.L.: Reconstruction of the human cerebral cortex from magnetic resonance images. IEEE Trans. Med. Imag. 18(6) (1999) 467-480

2. Kriegeskorte, N., Goebel, R.: An efficient algorithm for topologically correct segmentation of the cortical sheet in anatomical mr volumes. NeuroImage 14 (2001) 329-346

3. Dale, A.M., Fischl, B., Sereno, M.I.: Cortical surface-based analysis i: Segmentation and surface reconstruction. NeuroImage 9 (1999) 179-194

4. Fischl, B., Liu, A., Dale, M.: Automated mainfold surgery: Constructing geometrically accurate and topologically correct methods of the human cerebral cortex. IEEE Trans. Med. Imag. 20 (2001) 70-80

5. Shattuck, D., Leahy, M.: Automated graph-based analysis and correction of cortical volume topology. IEEE Trans. Med. Imag. 20(11) (2001) 1167-1177

6. Han, X., Xu, C., Neto, U., Prince, J.: Topology correction in brain cortex segmentation using a multiscale, graph-based algorithm. IEEE Trans. Med. Imag. 21(2) (2002) 109-121

7. Wood, Z., Hoppe, H., Desbrun, M., schroeder, P.: Removing excess topology from isosurfaces. ACM Transactions on graphics 23 (2004) 190-208

8. Bertrand, G.: Simple points, topological numbers and geodesic neighborhoods in cubic grids. Pattern Recognition Letters 15 (1994) 1028-1032 